\title{
LA SEDUCCIÓN DE LAS LENGUAS Y LOS JÓVENES (2a PARTE): ESTUDIO SOBRE MUDAS LINGÜÍSTICAS Y NEOHABLANTES
}

\author{
THE SEDUCTIVENESS OF LANGUAGES FOR THE YOUNG PEOPLE (PART 2): A \\ STUDY OF LANGUAGE SHIFT AND NEW SPEAKERS
}

\author{
Raquel Casesnoves Ferrer \\ Universitat Pompeu Fabra \\ raquel.casesnoves@upf.edu
}

Recibido: $07 / 05 / 2015$

Aceptado: 28/01/2016

\begin{abstract}
Resumen
Recientemente en el mundo académico se ha difundido y popularizado la noción de neohablantes. Hace algunos años estudiamos el poder de atracción que el valenciano, en proceso de normalización desde la década de los 80 del siglo pasado, y el castellano, ejercían sobre los jóvenes valencianos. En este artículo nos proponemos, además de contribuir a difundir los últimos datos procedentes de encuestas y censos lingüísticos que no han sido ampliamente publicados, retomar el tema de la seducción del valenciano para averiguar la posible influencia de la edad, además del lugar de nacimiento (y residencia). Los resultados muestran el retroceso imparable del valenciano entre la sociedad valenciana por una parte y, por otra, la importancia de la zona de residencia en las mudas lingüísticas de los jóvenes.

PALABRAS CLAVE: neohablantes, muda lingüística, valenciano, catalán, País Valenciano, jóvenes.
\end{abstract}

\begin{abstract}
Recently, the notion of Newspeakers has been spread and popularized in academia. Some years ago, we studied the power of attraction of the Valencian language, in a process of normalization since the 80 s of last century, and Spanish, on the youngsters in the Valencian Country. The aim of this article, besides contributing to disseminate the latest data from surveys and linguistic censuses that have not been widely published, is to revisit the issue of seduction of Valencian to find out the influence of age and place of birth (and residence). The results show the unstoppable decline of Valencian among the Valencian society, on the one hand, and, on the other, the importance of the area of residence in the linguistic mudes of young people.

KEYWORDS: Newspeakers, linguistic mude, Valencian, Catalan, Valencian Country, Youngsters.
\end{abstract}

Para citar este artículo / To cite this article: Casesnoves Ferrer, Raquel (2016). La seducción de las lenguas y los jóvenes ( $2^{\mathrm{a}}$ parte): estudio sobre mudas lingüísticas y neohablantes. ELUA, 30: 43-60. doi: 10.14198/ELUA2016.30.02

Enlace / Link: http://dx.doi.org/10.14198/ELUA2016.30.02 


\section{Introducción ${ }^{1}$}

El año 2008 se conmemoraba el vigésimo quinto aniversario de la entrada en vigor de la Ley de uso y enseñanza del valenciano (eufemismo de normalización lingüística) en el País Valenciano con la celebración de diversos actos académicos, publicaciones oficiales que se congratulaban de los logros conseguidos y publicaciones extraoficiales muy críticas con el desarrollo de la política lingüística. A mi modo, y ajena al ámbito mediático y oficial, celebré este hecho transcendental para el catalán presentando en un Congreso nacional un trabajo, publicado con posterioridad (Casesnoves, 2010), sobre la capacidad de atracción que ejercía el valenciano ${ }^{2}$ entre los jóvenes castellanohablantes. También allí mostraba los principales resultados de una encuesta oficial sobre los usos lingüísticos de la población valenciana (AVL, 2005) que, a parte de constatar que cada vez se hablaba menos valenciano, seguía reiterando el clásico patrón de comportamiento diglósico. De los resultados que aportaba mi estudio, basado en una muestra de 320 estudiantes de la ciudad de Valencia y de Xàtiva (capital de una de las comarcas centrales situada en el interior y a $54 \mathrm{~km}$ de la capital), destacaré la importancia de la zona de residencia en la elección de lengua y, como era de esperar, en el diferente poder que tienen las lenguas para atraer e incorporar nuevos hablantes.

En el 2015 no celebramos ningún aniversario lingüístico, pero es un año de elecciones municipales y, en algunas comunidades, autonómicas e incluso plebiscitarias. Las consecuencias lingüísticas que el poder político tiene en el ámbito del municipio no ha merecido ningún interés porque las leyes emanan de jerarquías de poder superiores, pero es evidente que esto afecta el paisaje lingüístico del municipio, la partida presupuestaria destinada a fomentar el valenciano (si existe) y, por ende, la vida diaria del ciudadano. Aunque es evidente que este artículo no forma parte de la campaña política de ningún partido, no está demás insistir en un hecho que nos parece obvio, además de totalmente ajeno a nuestro interés. Si bien la investigación lingüística no puede estar al margen de la realidad social y política, también lo es que de entrada no debe hacer bandera de ninguna ideología. Nuestro artículo no tiene más pretensión que contribuir a la divulgación de los datos de una encuesta del 2010 (SIES, online) y de un censo lingüístico del 2011 (IVE, online), realizados ambos con dinero público y que no han merecido ninguna publicación oficial, además de sacar a la luz los resultados de nuestra propia encuesta. Tal vez la información y el conocimiento no nos haga libres, como afirmaba Sócrates, pero al menos que sea la base que nos permita tomar decisiones.

El título del artículo no es baladí, puesto que la referencia a una segunda parte responde a nuestro objetivo de continuar investigando sobre el poder de atracción que tienen el castellano y el valenciano en los jóvenes y en averiguar la posible influencia de la edad, además del lugar de nacimiento (y residencia). La nueva encuesta, que es la que permite la comparación entre los dos momentos, recoge los datos demográficos y lingüísticos de casi 200 estudiantes

1 Quisiera expresar mis más sinceros agradecimientos al Institut Universitari de Lingüística Aplicada de la Universitat Pompeu Fabra por seguir acogiéndome en sus instalaciones y poder conservar mi espacio durante todo este tiempo de dificultades laborales. Y, como siempre, al profesor David Sankoff, por su constante y persistente ayuda. Parte de los resultados que aquí se presentan forman parte de un Proyecto de Investigación Nacional financiado por el Ministerio de Economía y Competitividad del Gobierno de España (FFI2010-16066).

2 En este artículo usamos el término "valenciano" para referirnos al catalán que se habla en el País Valenciano. Queremos dejar claro que, al desechar el término "catalán", denominación académica internacional, no negamos la filiciación genética entre valenciano y catalán. Nuestra elección coincide con la denominación oficial así como tradicional y popularmente reconocida. 
universitarios cuatro años después que se realizara la primera, en el año 2008, a una muestra de 320 estudiantes de secundaria. Las diferencias metodológicas entre las dos encuestas, que afectan directamente el estudio que aquí llevamos a cabo, serán explicadas con detalle tras presentar los principales resultados oficiales sobre el conocimiento del valenciano y los usos lingüísticos de la población valenciana. Después de analizar nuestros propios datos reflexionaremos sobre las consecuencias sociolingüísticas del poder de atracción que tienen las dos lenguas que conviven diariamente en el País Valenciano, especialmente sobre el valenciano, por ser la lengua más desprotegida y vulnerable.

\section{El conocimiento del valenciano en el siglo XXI}

El año 2011 se llevó a cabo el Censo de Población y Vivienda que permite, entre otros temas, conocer las características demográficas y sociales de la población (sexo, edad, estudios...). Realizado de manera oficial cada diez años desde 1900, no tenemos ninguna información lingüística hasta llegar al período democrático actual. Teniendo en cuenta que el Padrón Municipal de $1986^{3}$ fue el primero en incluir en las comunidades bilingües algunas preguntas lingüísticas, contamos con información sobre un período de casi 30 años. Una información muy limitada que permite saber la cifra de población que es capaz de entender, hablar, leer y escribir la otra lengua oficial (utilizando terminología constitucional). La cuestión se plantea en términos dicotómicos (Sí /No) y la persona que rellena el cuestionario juzgará su propio conocimiento así como el del resto de miembros de la familia. Podemos dudar de su validez, puesto que es lógico pensar que el conocimiento de una lengua debe medirse con otros parámetros, pero no ponemos en tela de juicio ni su fiabilidad ni su gran aportación histórica, que permite obtener longitudinalmente unos datos comparables.

La figura 1 ilustra el porcentaje de individuos que, de cada grupo de edad, es capaz de entender y de hablar valenciano (conocimiento oral pasivo y activo respectivamente) desde el año 1986 hasta el 2011. Hemos sombreado la franja de edad de los jóvenes porque, además de ser los que más han aumentado sus niveles de competencia con los años, nuestro estudio está centrado en ellos.

La distribución del conocimiento que la línea del año 1986 perfilaba, con un incremento paulatino de la competencia lingüística con la edad, es típica de lenguas que están en proceso de substitución avanzado y en peligro claro de extinción (los jóvenes ya no conocen una lengua que acabará desapareciendo con las viejas generaciones). Los efectos de la etapa democrática, con nuevas leyes que regulan el uso de la lengua y, sobre todo, su introducción en el currículum escolar, son enormemente rápidos. En tan sólo cinco años las líneas empezaron a invertirse y en 1991 el nivel de capacidad para hablar valenciano de los jóvenes en edad escolar (10-19 años) iguala al de los de edad más avanzada. Y esta nueva tendencia que parece alterar profundamente la relación social entre el valenciano y el castellano no ha dejado, desde entonces y de forma muy clara, de acentuarse.

3 El Padrón Municipal constituye el registro administrativo, dependiente de cada ayuntamiento, donde constan los vecinos de la localidad y se actualiza el 1 de enero de cada año. Hasta el año 1996, en el que se modificó la normativa y se estableció un nuevo sistema de gestión, coordinación y centralización por parte del Instituto Nacional de Estadística, la Revisión y Renovación del Padrón municipal se realizaba quinquenalmente, momento en el que se hacían públicas las cifras oficiales. 


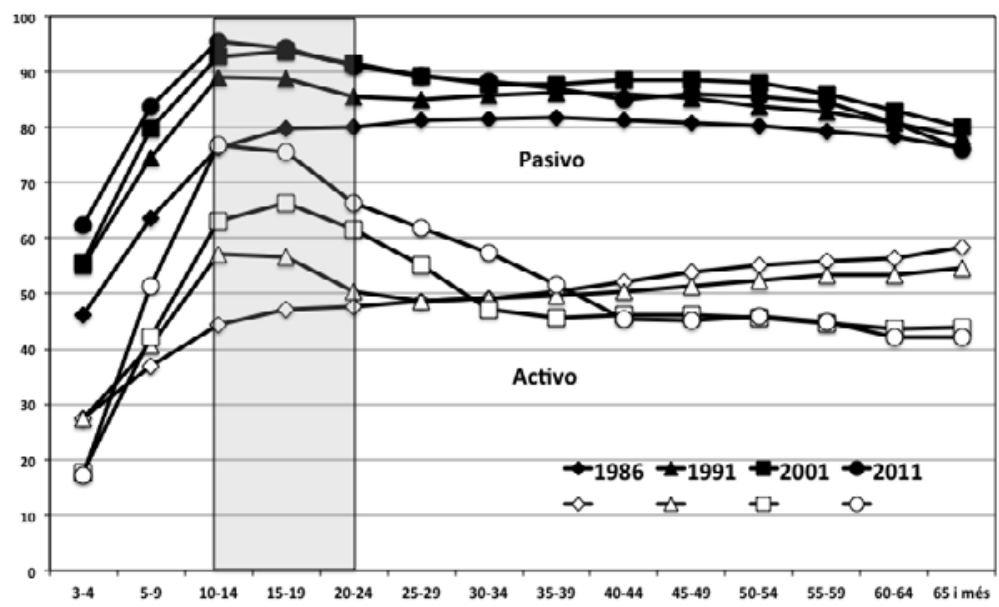

Gráfico 1. Evolución del conocimiento oral del valenciano según los Censos de Población.

Concisa y claramente, la escuela ha logrado que los jóvenes adquieran el conocimiento necesario para poder hablar valenciano, alejando aparentemente las amenazas de extinción.

\section{Los usos lingüísticos de la población valenciana: evolución y diferencias territoriales}

Las fuentes que nos sirven de base para examinar ya no tanto las declaraciones sobre si se sabe hablar o no valenciano que refleja el gráfico 1 como sobre si se habla o no en determinadas situaciones, y en que grado se habla junto con el castellano, son igualmente oficiales pero de ámbito autonómico. Desde que inició su actividad a principios de los años 80 del siglo pasado, el Servicio de investigaciones y estudios sociolingüísticas (SIES) de la Generalitat Valenciana ${ }^{4}$, ha realizado numerosas encuestas sobre el uso del valenciano en diferentes ámbitos (la radio, la administración, las superficies comerciales, empresas y comercios), en grandes ciudades (Valencia, Alicante, Castellón, Elche) así como en todo el territorio. En estas últimas, que son las que aquí nos interesan, se pregunta a una muestra representativa de la población si habla siempre, generalmente, más o indistintamente valenciano y castellano en una serie de diferentes situaciones supuestamente típicas. La gran ventaja que ofrecen estas encuestas es que siempre se ha seguido la misma metodología, por lo que los resultados son perfectamente comparables. En el gráfico 2 hemos ilustrado el uso preferente (adición de las respuestas siempre y generalmente) del valenciano en casa, con los amigos, en pequeños comercios, en grandes superficies y con los viandantes de la población valenciana que reside en la zona valencianohablante. Se excluyen pues las 10 comarcas que integran la zona donde históricamente se ha hablado castellano y de las que no tenemos más datos que el conocimiento del valenciano.

Hay que destacar, en primer lugar, la gran diferencia entre el porcentaje de población valenciana que declara saber hablar valenciano (gráfico 1), que el año 2011 se situaba alrededor

4 Lamentamos profundamente la reciente desaparición de este servicio público que se añade a la lista de servicios en valenciano que cesan de ejercer su función pública, como la RTTV. 
del 50\%, y el de aquella que dice hablarlo: un tercio según la encuesta del año 2010, y en el contexto que tradicionalmente siempre se ha hablado con mayor frecuencia, que es el ámbito familiar. La otra gran diferencia, que invalida la mayoría de las conclusiones que de la figura 1 hemos extraído, se encuentra en la dirección de esas líneas que ilustran las figuras y que marca dos tendencias opuestas: una positiva, puesto que el conocimiento de la población ha ido aumentando con los años, como hemos explicado, y otra, en cambio, negativa, ya que esa misma población que declara que sabe hablar valenciano también dice que prefiere hablar castellano.

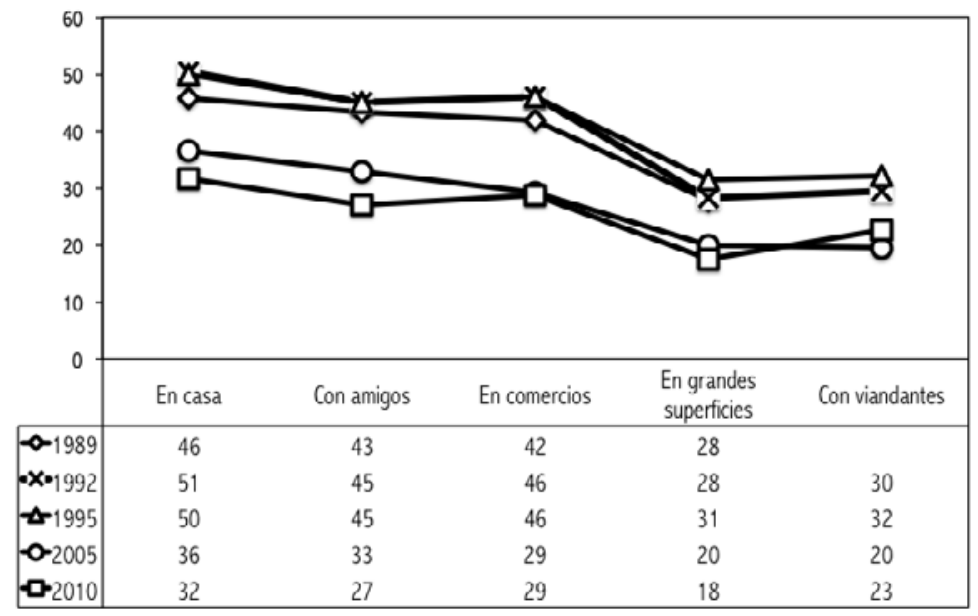

Gráfico 2. Evolución del uso del valenciano en diferentes situaciones entre 1989 y 2010.

El cambio de tendencia, cuantitativamente importante, se produce entre 1995 y 2005 . El retroceso, que sobrepasa el 10\% en todos los ámbitos considerados, es especialmente grave en el familiar, por su incidencia en el uso del valenciano fuera de este contexto (Castelló, 2006; Montoya, 2008; Pradilla, 2008; Casesnoves, 2012). Por otra parte, el comportamiento de la población sigue anclado en patrones típicos de situaciones diglósicas, con frecuencias de uso más altas en los contextos más privados e íntimos, como el hogar, y con personas conocidas, como los amigos, y frecuencias más bajas en ámbitos impersonales, con desconocidos, o asociados a grandes empresas y centros comerciales.

Los datos generales sobre el uso del valenciano esconden importantes diferencias territoriales. Por eso, el SIES ha tenido siempre en cuenta la influencia de la comarca de residencia en el uso de la lengua distinguiendo cinco regiones dentro de la zona históricamente valencianohablante (véase el mapa comarcal más abajo): la región de Valencia (la Ribera Baixa, la Ribera Alta, la Costera, el Camp de Morvedre y el Camp de Túria); la región de Alcoi-Gandia (la Marina Alta, el Comtat, l'Alcoià, la Vall d'Albaida y la Safor); la región de Castellón (la Plana Alta, la Plana Baixa, l'Alcalatén, l'Alt Maestrat, el Baix Maestrat y els Ports); la región de la ciudad de València y área metropolitana (Valencia, l'Horta Nord, l'Horta Sud y l'Horta Oest) y la región d'Alacant (el Baix Vinalopó, la Marina Baixa, les Valls del Vinalopó y l'Alacantí). Para poder observar bien estas diferencias el gráfico 3 ilustra el uso del valenciano en el ámbito familiar a través de los años según estas cinco regiones, ordenadas de izquierda a derecha según el nivel más alto de uso del valenciano en el año de partida, el 1989. 


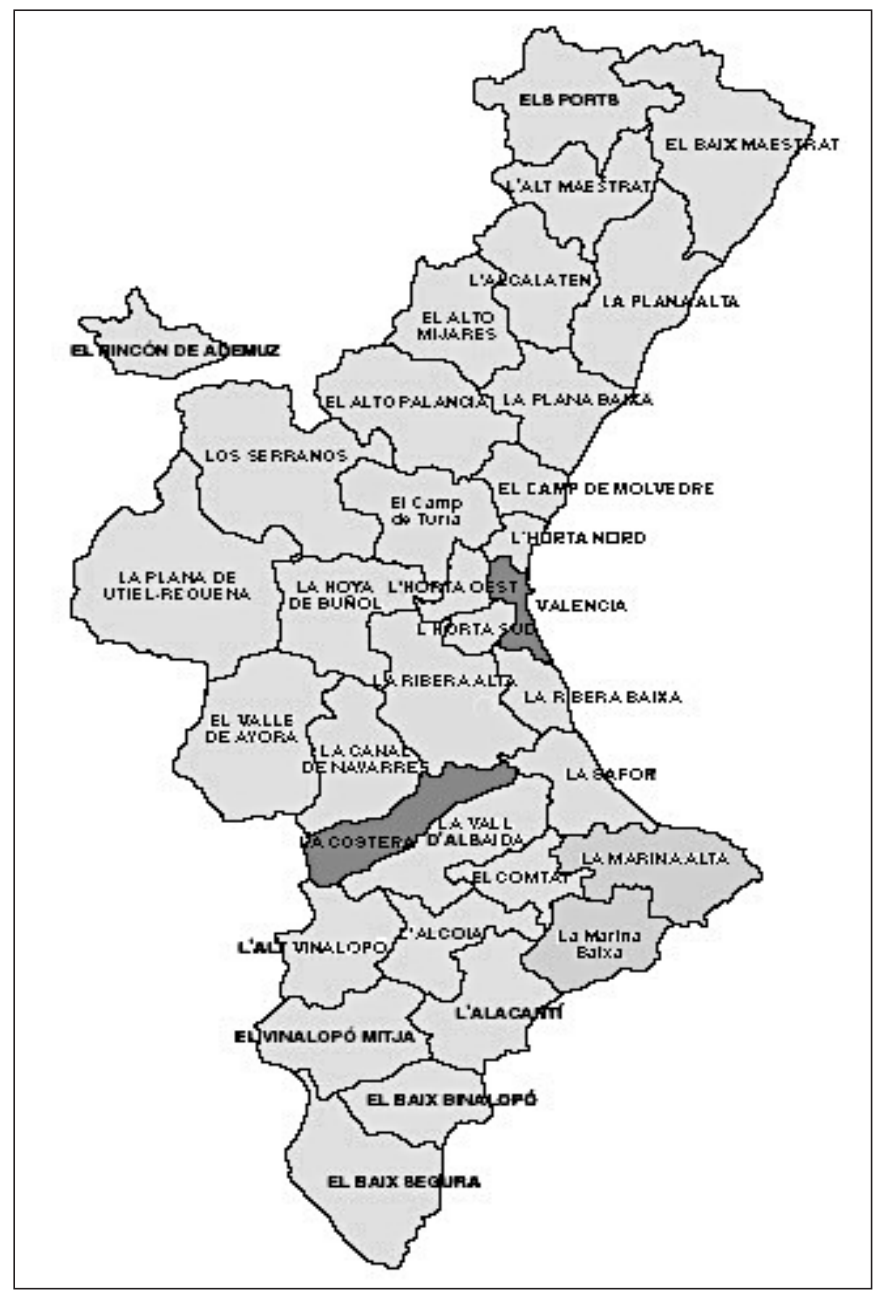

Mapa 1. Las comarcas del País Valenciano.

Las comarcas de las regiones de Alcoi-Gandia y Valencia destacan por ser las que tienen más valencianohablantes, entre un 55 y un $60 \%$ respectivamente, según datos de la encuesta del año 2010. En la región de Alicante, donde no llegaba a un tercio de la población la que declaraba hablar valenciano en casa a principios de los 90 , había un 15\% menos de hablantes, como en el área metropolitana de Valencia. En Castellón, el retroceso alcanza el 20\%, el dato más elevado. Interesa destacar que es en las comarcas centrales del País Valenciano, históricamente cohesionadas desde un punto de vista económico y cultural, donde más se habla valenciano y donde, en el período analizado, menos se pierde.

Resumiendo, conocimiento y uso van por caminos distintos y en direcciones opuestas. No hay nada nuevo en esta afirmación que no se haya constatado mucho antes. Esta diferencia entre competencia y uso, acentuada en el caso de los escolares, se ha denominado de maneras diferentes -latinización (Ferrando, 1991), extranjerización o asignaturización 


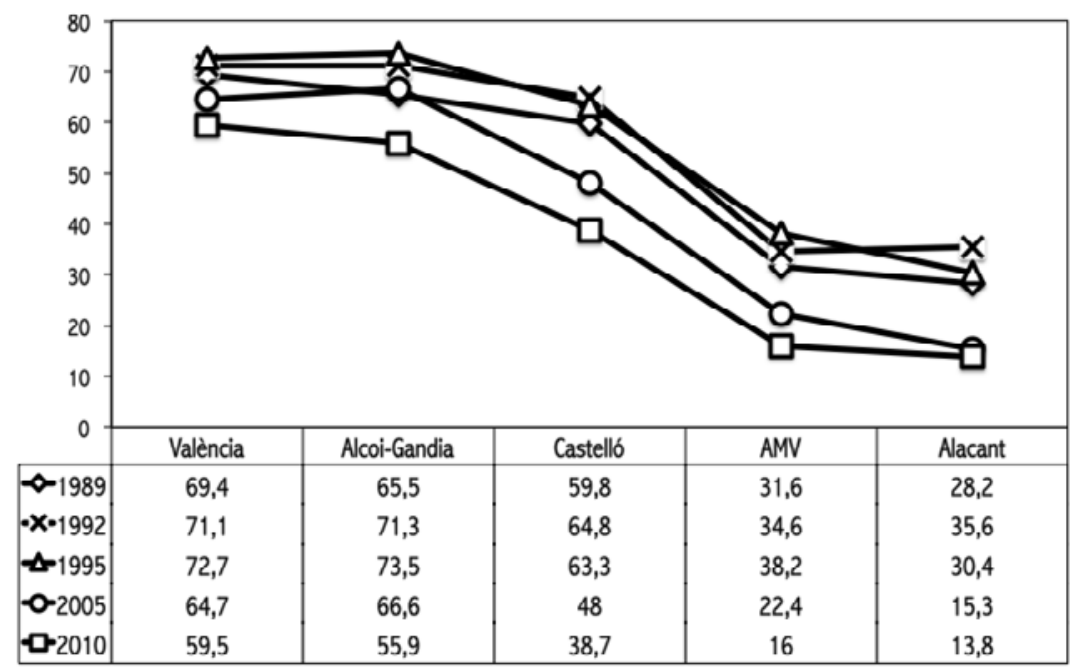

Gráfico 3. Evolución del uso del valenciano en el ámbito familiar entre 1989 y 2010 según la región de residencia.

(Aparici et al., 1994; Aguilar, 2001) e, incluso, irlandización (Colom, 1998; Bodoque, 2009) - para referirse al alejamiento del valenciano en la vida de los jóvenes. Lo que sorprende es que siga perpetuándose esta contradicción que indica un proceso imparable de substitución lingüística, cuando lo que deberíamos encontrar es una corrección y un uso más elevado del valenciano que fuera a la par con el conocimiento. Además, otros autores (Montoya y Mas, 2011) han encontrado, aunque a pequeña escala, un fenómeno nuevo que refiere a los nuevos hablantes ${ }^{5}$ o personas que hablan habitualmente una lengua, el valenciano en este caso, que no es su lengua familiar (el castellano u otra). Esta capacidad de atraer nuevos hablantes que tradicionalmente ha tenido el castellano y que genera que cada vez haya menos valencianohablantes parecería ser pues que también la podría estar ejerciendo el valenciano. $\mathrm{Y}$ es esto precisamente lo que nos interesa explorar. ¿Seduce en alguna medida el valenciano a los jóvenes castellanohablantes? O, al contrario, ¿sigue teniendo el castellano más poder seductor que el valenciano? ¿Y en que medida las diferencias territoriales interfieren en estos procesos?

En lo que sigue intentamos responder a estas preguntas basándonos en dos estudios realizados en dos momentos diferentes, 2008 y 2012, en dos momentos de la vida de los jóvenes especialmente relevantes en los cambios de comportamiento, en el instituto y en la universidad, y en dos zonas territoriales donde las diferencias de uso del valenciano son muy importantes: la región de Valencia y la ciudad de Valencia.

5 De unos años acá, la realización de simposios internacionales sobre los New speakers o Neohablantes (2012 y 2014) así como la gran cantidad de trabajos sobre este tema (la revista Caplletra ya le dedicó un monográfico el año 2012, núm. 53, y más recientemente lo ha hecho International Journal of the Sociology of Languages, núm. 231) muestran el gran interés suscitado. 


\section{Aspectos metodológicos}

Los usos lingüísticos que consideramos para poder analizar la trayectoria lingüística de los estudiantes y descubrir así el poder seductor de las lenguas parten de la primera lengua o de inicio y acaban en la lengua de uso en el momento de la encuesta.

En este apartado explicaré las principales diferencias metodológicas que existen entre las dos encuestas (de ahora en adelante E-2008 y E-2012 según el año de realización), diferencias que afectan tanto las características sociodemográficas de la muestra, como el cuestionario o el modus operandi de la recogida de datos.

\subsection{Procedimiento}

La principal diferencia en la manera de proceder para acceder a la información consiste en la utilización de las nuevas tecnologías el año 2012. Así, mientras que en la E-2008 estuvimos presentes en cada aula de los diferentes centros de secundaria en el momento en que los estudiantes contestaban por escrito a las preguntas del cuestionario, en la E-2012, los universitarios podían acceder al cuestionario a través de internet y no estuvimos siempre presentes $^{6}$. A pesar de esto, sólo el $18 \%$ de los datos proceden de estudiantes que completaron la encuesta fuera del aula de la Universidad.

En ambas encuestas las muestras no son ni pretendían ser representativas de la población total de estudiantes. El azar, por otra parte, tiene un punto más de importancia en la E-2012 al no poder controlar los estudiantes que accedían al cuestionario fuera del aula.

\subsection{El cuestionario sociolingüístico}

En relación con el cuestionario y dejando de lado que el total de preguntas es muy diferente (32 en E-2008 y 67 en E-2012), lo que aquí resulta pertinente, a efectos comparativos, son las preguntas específicas que recogen los datos sobre la lengua inicial y la lengua habitual. Como hicimos en el trabajo anterior (supra cit.) siguiendo a Fabá (2005) y a Villaverde (2007), consideramos que la lengua inicial corresponde a la lengua que hablan con los progenitores. Exceptuando la opción de respuesta siempre en otra lengua ${ }^{7}$, el resto eran exactamente las mismas en ambas encuestas (vid. Tabla 1). Para poder analizar luego los datos, las respuestas se clasificaron en tres: valenciano $(1,2$ y 7$)$, castellano $(4,5$ y 8$)$ y las dos por igual (3).

En relación con la lengua habitual o lengua de uso predominante en el momento de la encuesta, existe una importante diferencia entre los dos estudios y es que en la E-2008 se incluía una pregunta sobre la lengua de identificación (¿Puedes indicar cuál es tu lengua?) ausente en la E-2012. En su lugar se incluyó una cuestión sobre la lengua habitual (¿Qué lengua usas más a menudo?) por ser más explícita. Aunque las opciones de respuesta son las mismas (Tabla 1), puesto que en la opción de Otros casos en la E-2008 se respondió de hecho el uso indistinto de las dos lenguas, tendremos que tener en mente esta diferencia cuando comparemos los resultados de ambas encuestas.

$6 \quad$ El primer contacto con los profesores se hizo a través de las listas de distribución de correo electrónico. En el mensaje se pedía ayuda para difundir la encuesta entre sus alumnos, cediéndonos un tiempo prudencial en el grupo de clase o para publicitar nuestro proyecto y el sitio internet desde donde podían completarla.

7 Hay que aclarar que estas respuestas no se tuvieron en cuenta por su escasa frecuencia. 


\begin{tabular}{l|l}
\hline \multicolumn{1}{c|}{ Lengua inicial } & \multicolumn{1}{|c}{ Lengua de identificación/habitual } \\
\hline $\begin{array}{l}\text { 1. Siempre valenciano } \\
\text { 2. Más en valenciano que en castellano } \\
\text { 3. Indistintamente, valenciano y castellano }\end{array}$ & 2. Valenciano \\
4. Más en castellano que en valenciano \\
$\begin{array}{l}\text { 5. Siempre en castellano } \\
\text { 6. En otra lengua y en valenciano }\end{array}$ \\
$\begin{array}{l}\text { 7. En otra lengua y en castellano } \\
\text { 8. Otras dos (E-2012) }\end{array}$ \\
\hline
\end{tabular}

Tabla 1. Opciones de respuesta de lengua inicial y lengua de identificación/habitual.

\subsection{La edad}

Los jóvenes, como queda dicho, constituyen el objeto de estudio de las dos encuestas que realizamos. Sin embargo, la E-2008 se centra en los estudiantes de secundaria, mientras que la E-2012 lo hace en los que cursan grados. Diecisiete es la media de edad de los jóvenes de instituto y 21 la de los universitarios. La diferencia de edad coincide con la diferencia en la que se realizaron las encuestas, cuatro, por lo que, aun tratándose de jóvenes diferentes pertenecen, de hecho, a la misma generación.

La importancia de la edad en nuestro estudio, centrado en el cambio de comportamiento lingüístico, es fundamental. Y eso porque el cambio o muda lingüística (Pujolar et al. 2014) suele ocurrir en momentos decisivos de la vida, de los cuales destacan el inicio del instituto y de la carrera universitaria (Marí, 2012). En este sentido nuestro trabajo nos podría ayudar a comprobar que momento de los dos es mas propicio al cambio lingüístico.

\subsection{Origen y residencia}

El estudio del año 2008 tenía como objetivo fundamental analizar los usos lingüísticos de los estudiantes de secundaria de la gran urbe y capital del País, la ciudad de Valencia, y después compararlos con los de una ciudad capital de comarca, Xàtiva. En el año 2012 nos planteamos explorar el comportamiento de un colectivo de jóvenes poco estudiado, el de universitarios. Nos centramos en los estudiantes de la ciudad de Valencia porque es donde se encuentran localizadas las principales universidades públicas y el objetivo no era tanto la residencia en la capital como el hecho de ser estudiante universitario 9 . Por ese motivo no disponemos de información alguna sobre la residencia de los jóvenes de E-2012, pero sí sobre el lugar de nacimiento, que será la variable que utilizaremos para comparar las muestras de ambas encuestas. El gráfico 4 muestra la distribución de los estudiantes de

8 El concepto de muda lingüística enfatiza el papel del hablante en la decisión de modificar sus normas de uso habituales haciendo hincapié también en el momento en que se realiza este cambio en su trayectoria vital.

9 De la encuesta de universitarios quedaron excluidos, de entrada, los estudiantes de intercambio, tipo Erasmus. 


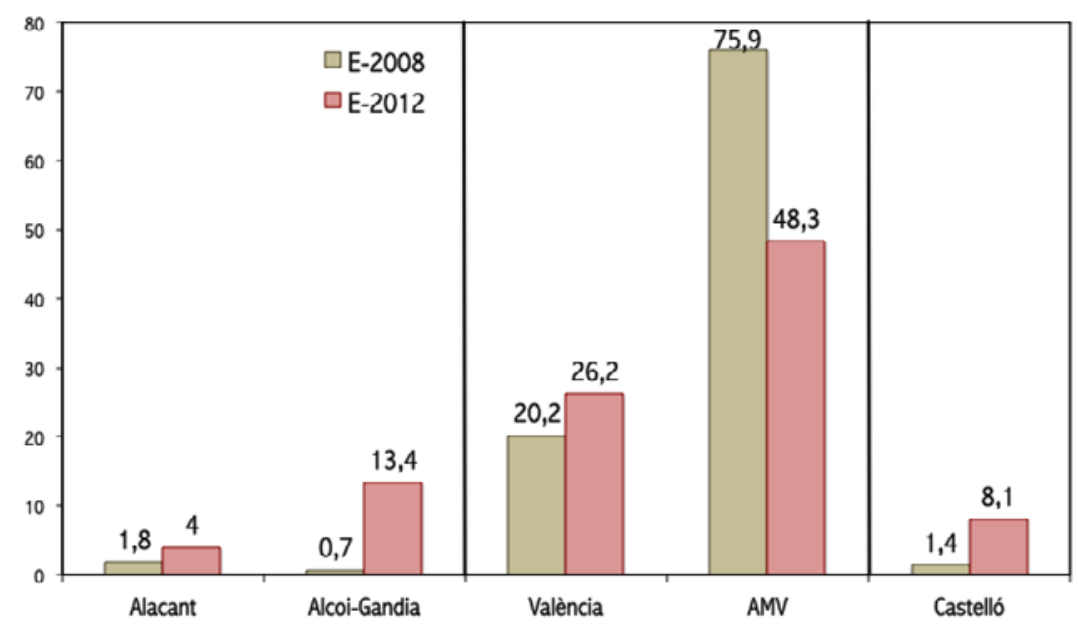

Gráfico 4. Distribución de las muestras según la región valencianohablante de nacimiento.

ambas encuestas según las 5 regiones valencianohablantes a las que hemos aludido en la sección anterior. Quedan excluidos de este estudio pues aquellos estudiantes que nacieron en una comarca castellanohablante, en otra parte de España o en el extranjero, además de aquellos que respondieron con un simple Valencia $^{10}$ (39 en E-2008 y 40 en E-2012).

Como podíamos esperar, la clasificación de los estudiantes universitarios (E-2012) en las diferentes regiones está más repartida que en la de los estudiantes de secundaria (E-2008), puesto que es lógico suponer que los desplazamientos a la metrópoli son mucho más numerosos cuando se cursan estudios universitarios que secundarios. El 20\% de la región de Valencia de E-2008 corresponde casi en su totalidad a estudiantes de Xàtiva, mientras que el 26\% de E-2012 lo hace a estudiantes que nacieron en diferentes comarcas. Como vemos, el grueso de las muestras se concentra en estas dos regiones que son las que compararemos en la sección de análisis.

\subsection{Relación entre lengua inicial (L1) y lengua habitual/identificación (L2)}

Para observar la trayectoria lingüística de los informantes relacionamos la lengua inicial (L1) con la lengua de identificación o habitual (L2) construyendo una nueva variable a partir de estas dos. De esta forma obtendremos una clasificación de lo que llamaremos "la trayectoria lingüística" de los estudiantes. La tabla 2 muestra todos los casos que resultan de combinar las opciones de respuesta de la L1 y la L2. Los casos de no mutación, en que la L1 y la L2 coinciden, se corresponden con el Caso 1 para el valenciano, el Caso 5 para el castellano y el Caso 9 para las dos, a los que, siguiendo con la terminología empleada en el trabajo anterior, denominaremos homolingües valencianos, homolingües castellanos y homolingües bilingües respectivamente. En el resto de casos se produce siempre una

10 Este término es ambiguo porque puede hacer referencia tanto a la provincia como a la ciudad de Valencia. 
mutación que es lo que permite medir el cambio favorable al valenciano (casos 2, 3 y 8) o al castellano (casos 4, 6 y 7) y a los que denominaremos mutantes valencianos y mutantes castellanos respectivamente.

\begin{tabular}{lll}
\hline & L1 & L2 \\
\hline Caso 1 & Valenciano & Valenciano \\
Caso 2 & Castellano & Valenciano \\
Caso 3 & Las dos & Valenciano \\
Caso 4 & Valenciano & Castellano \\
Caso 5 & Castellano & Castellano \\
Caso 6 & Las dos & Castellano \\
Caso 7 & Valenciano & Las dos \\
Caso 8 & Castellano & Las dos \\
Caso 9 & Las dos & Las dos \\
\hline
\end{tabular}

Tabla 2. Casos resultantes de combinar las opciones de respuesta de L1 y L2

\subsection{El poder de atracción del valenciano: los neohablantes}

Los casos mutantes que resultan de la clasificación de los estudiantes según su trayectoria lingüística presentada en la sección anterior no permite descubrir los individuos que han introducido una nueva lengua en su repertorio lingüístico más allá de los casos 7 y 8 , ya que en el resto de casos lo que se produce en realidad es el abandono de una por otra (casos 2, 3, 4, 6).

Siguiendo a Ramallo (2013), consideramos neohablante aquella persona que aprende una lengua minoritaria o recesiva (aquí el valenciano) fuera del contexto familiar y que en un momento dado de su vida desplaza su lengua materna (el castellano) y pasan a hablarla de manera prioritaria. Se trata de una caracterización restrictiva (puesto que deja fuera a los individuos que la hablan de manera menos frecuente $u$ ocasionalmente) que seguimos en este trabajo al interpretar que las respuestas a la lengua con la que se identifican u hablan de manera habitual los estudiantes responde a aquella que usan más a menudo.

A efectos prácticos esta cualificación implica considerar neohablantes a los estudiantes que tuvieron tanto el castellano como el castellano y el valenciano como lengua inicial y que declaran identificarse con o hablar habitualmente el valenciano (casos 2 y 3 , quedando excluido el caso 8 , considerado en la tabla 2 como mutante al valenciano).

\section{Resultados}

Antes de presentar las diferentes trayectorias lingüísticas de los estudiantes y de descubrir la existencia o no de neohablantes, es conveniente saber la distribución de los estudiantes según la lengua inicial y la L2 y las diferencias territoriales y temporales que existen. En los gráficos que siguen distinguiremos pues, tanto la región de nacimiento como el año de encuesta. 

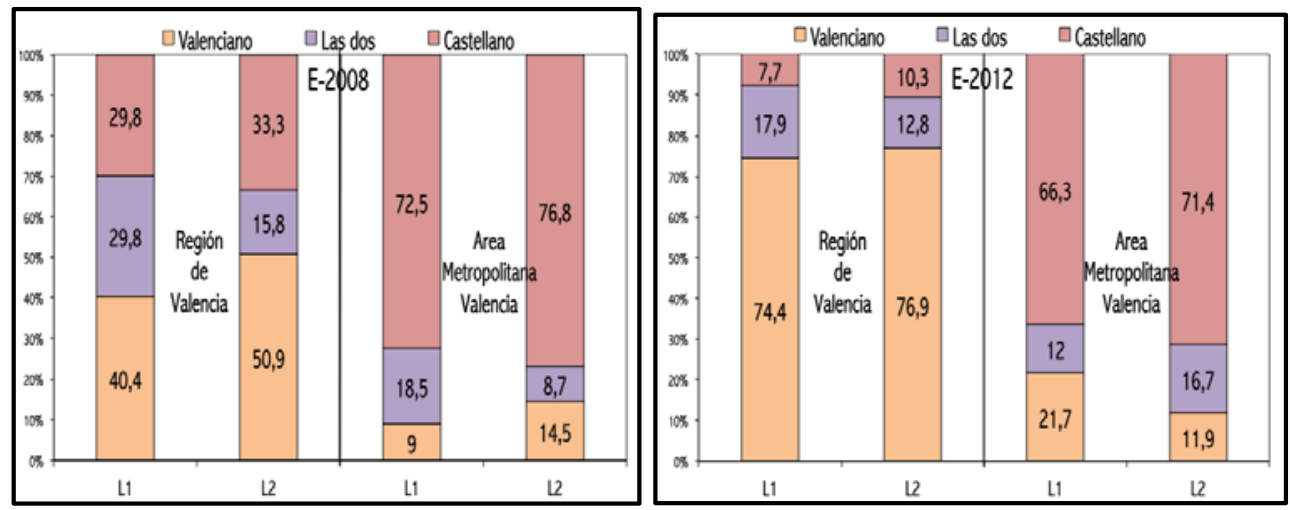

Gráfico 5. Distribución de las muestras según la L1 y la L2 y lugar de nacimiento.

La proporción de valencianohablantes iniciales en las dos encuestas es muy diferente, puesto que aumentan considerablemente el año 2012, y eso en las dos regiones, Valencia y el Área metropolitana. Un resultado que contradice los datos presentados más arriba, generales a toda la población valenciana (vid. gráfico 3) y que indican claramente un retroceso del valenciano en el ámbito familiar. Y con todo, esta desviación no nos parece importante para nuestro interés, ya que nuestro objetivo no es comparar datos difícilmente contrastables $^{11}$.

Nos corresponde destacar las tendencias de uso en los dos momentos, similares aunque en proporciones diferentes sólo en la región de Valencia, donde el año 2008, en el paso de la L1 a la L2, el valenciano ganó un $11 \%$ de estudiantes (del 40\% al 51\%) y el 2012 un $2{ }^{\prime} 5 \%$. Este cambio no se hace en detrimento del castellano, que también aumenta (del $30 \%$ al $33 \%$ en la E-2008 y del 8\% al 10\% en la E-2012), sino de las dos. En la AMV, sin embargo, hay una diferencia temporal muy significativa, ya que la proporción de estudiantes con L2 valenciano es más alta que los que lo tienen como L1 (14,5\% y $9 \%$ respectivamente) el año 2008 pero no así en el 2012, que baja 10 puntos (del 22\% al 12\%). Este retroceso se compensa con el progreso del castellano y de las dos, que pasan del $66 \%$ al $71 \%$ y del 12 al $17 \%$ respectivamente.

Resumiendo, del gráfico 5 podríamos deducir que el poder de atracción que tiene el valenciano entre los estudiantes de secundaria se pierde entre los universitarios, en los que el cambio se realiza a favor del castellano. En la siguiente sección nos dedicamos a comprobarlo definitivamente.

\subsection{La trayectoria lingüística}

La trayectoria lingüística de los estudiantes, si a lo largo de su vida han pasado de hablar una lengua inicialmente con sus padres a hablar otra diferente y de manera prioritaria

11 En el estudio que publicamos hace algunos años intentamos explicar la desviación aludiendo a diferencias territoriales, ya que la región de Valencia en la E-2008 incluía sólo la ciudad de Xàtiva, que pudiera presentar niveles más bajos que el conjunto de la zona. 
después, nos ayudará a comprobar la frecuencia con la que cambian los jóvenes y en que lengua de las dos se ejerce este cambio en mayor proporción. De nuevo, asumiendo la premisa que los resultados generales esconderían diferencias territoriales de máxima importancia $^{12}$, mostramos los resultados según las dos regiones diferenciadas en este trabajo: la de Valencia y la metropolitana de la ciudad.
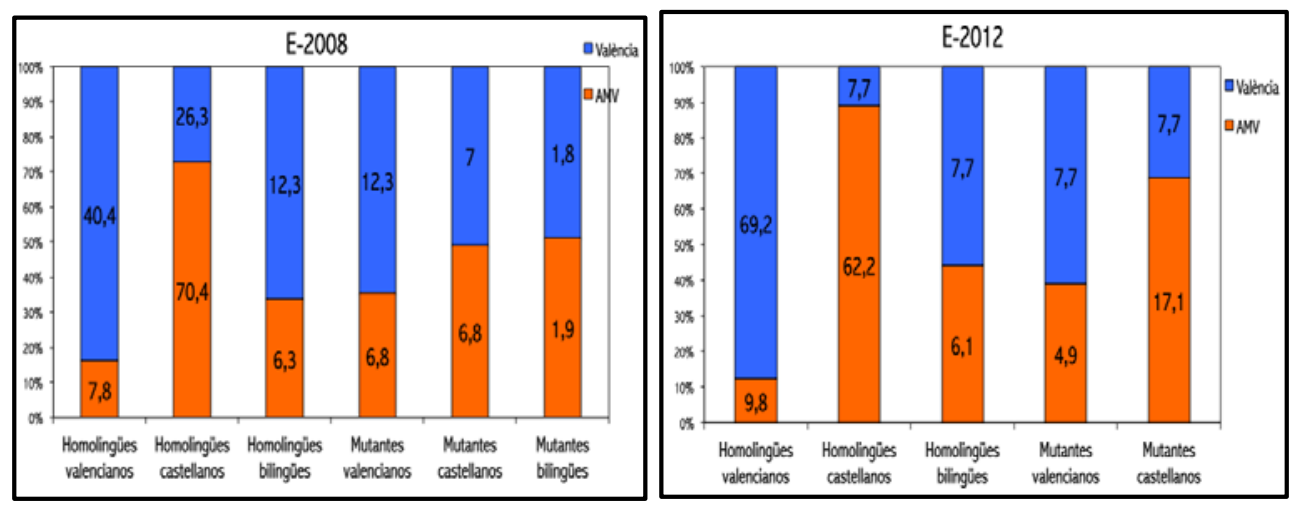

Gráfico 6. Distribución de los estudiantes según su trayectoria lingüística.

A primera vista podríamos pensar que se producen más cambios entre los estudiantes de secundaria (E-2008) que entre los universitarios (E-2012), simplemente por el hecho de que no hay casos de mutantes bilingües entre estos últimos. Centrándonos en la muestra del año 2008, las diferencias territoriales no parecen tener demasiado peso en los cambios de lengua de los jóvenes, puesto que la frecuencia total de mutantes en las dos regiones es bastante similar (15,5\% en la AMV y un poco más, $21 \%$, en la de Valencia). La diferencia fundamental, dejando de lado que los homolingües castellanos son mayoría entre los estudiantes originarios del AMV (el 70\% frente al 26\% de los de la región de Valencia), se encuentra en la frecuencia de la lengua hacia la que se realiza ese cambio de L1 a L2. Así, mientras que la proporción de estudiantes del AMV que mudan al valenciano es igual a la de los que mudan al castellano, un escaso 7\%, entre los jóvenes de la región de Valencia hay más casos de mutantes al valenciano (12\%) que al castellano (7\%). La diferencia no es muy grande $\mathrm{y}$, tratándose de una muestra pequeña y completamente aleatoria $(\mathrm{N}=49)$, es muy probable que los resultados se deban al azar.

La trayectoria lingüística de los universitarios (E-2012) no acaba de confirmar esta tendencia de cambio favorable al valenciano entre los estudiantes de Valencia, puesto que la proporción de mutantes es la misma en ambas lenguas (7,7\%). En cambio, aumentan considerablemente los jóvenes homolingües valencianos (un 30\% más que en E-2008) y descienden los homolingües castellanos (7\%), movimientos observables igualmente, aunque

12 Así han venido corroborándolo los resultados de los censos lingüísticos y de las encuestas sobre el conocimiento y uso del valenciano en el País. El criterio territorial, tanto por el hecho de residir en una u otra zona lingüística (la castellana o la valenciana) o en una ciudad con mayor o menor población (Ninyoles, 1992), como por habitar en una comarca de la zona valenciana u otra (Casesnoves, 2005 y Casesnoves, en prensa) es, sin lugar a dudas, el factor que más influye en los niveles de competencia y uso. 
en menores proporciones, entre los jóvenes del AMV (con un 8\% menos de homolingües castellanos y un 3\% más de homolingües valencianos). La diferencia fundamental entre los jóvenes de secundaria (E-2008) y los de universidad (E-2012) estriba en la proporción de mutantes al castellano, con un 10\% más entre estos últimos.

A pesar de que la proporción de homolingües, en valenciano o castellano, sea muy diferente en las dos muestras, no entraremos a intentar explicarla más de lo ya comentado en la sección anterior a propósito de la proporción de estudiantes que tuvieron el valenciano como lengua inicial. Nos interesa, eso sí, explicar las frecuencias de mutantes, ya que parecería que el cambio es mucho más probable hacia el castellano que hacia el valenciano, especialmente entre los universitarios del AMV. De manera que el cambio de lengua, que además es un poco más probable al iniciar una carrera universitaria, se realizaría en detrimento del valenciano. El castellano, además de ser lengua dominante, tendría el privilegio de seducir a jóvenes valencianohablantes. ¿Es esto realmente cierto o responde a una manipulación de datos engañosa?

\section{2. ¿Qué lengua tiene más poder de atracción entre los jóvenes?}

El tratamiento de los datos que resulta de considerar neohablantes a aquellos individuos que además de introducir una lengua en su repertorio lingüístico la hablan de manera habitual, tal como hemos explicado más arriba, nos permite descubrir con mayor facilidad el poder de atracción del valenciano y del castellano y, por ende, la proporción de verdaderos neohablantes (paso de L1 castellano a L2 valenciano y/o las dos) que discutiremos en las conclusiones. En los gráficos que siguen mostramos la lengua habitual o de identificación de los estudiantes según la lengua inicial que tuvieron, con el objetivo de que quede visualmente claro el poder de atracción de las dos lenguas.

Así, para los informantes de la E-2008 de L1 castellano que nacieron (y residen) en el Área Metropolitana de Valencia (Gráfico 7) el valenciano tiene un escaso atractivo porque la inmensa mayoría (el 96\%) siguen identificándose con el castellano. El castellano, al contrario, ejerce mucha más atracción entre los que tuvieron el valenciano como lengua inicial, ya que el $25 \%$ pasaron a identificarse con esta lengua. En cambio, entre los jóvenes de comarcas el poder de atracción del valenciano y del castellano es bastante similar: el 11\% de castellanohablantes iniciales se identifican con el valenciano, como el 10\% de valencianohablantes iniciales lo hace con el castellano.

Unos años más tarde, en cambio, para los universitarios de la región de Valencia el valenciano ya había perdido cualquier atractivo, puesto que ningún estudiante castellanohablante inicial decidiría luego hablar habitualmente valenciano. El castellano también pierde atractivo, pero aún llega a seducir a casi el $4 \%$ de valencianohablantes iniciales. Y en cuanto a los jóvenes del Área Metropolitana, mientras que el cambio de lengua al castellano se realiza en proporciones similares a como lo hacía entre los estudiantes de la E-2008 (26\%), el cambio al valenciano aumenta ligeramente (del 4 al 7\%).

En definitiva, a la pregunta sobre la lengua que atrae más a los jóvenes la respuesta es clara: el castellano, y eso independientemente de la edad, pero no de la zona donde se nació y, en su mayoría, también se reside. La comparación de las figuras 7 y 8 nos debería permitir, además, responder a la cuestión sobre si los jóvenes mudan con más facilidad cuando van al instituto o cuando ya están en la universidad. La respuesta en este caso de- 

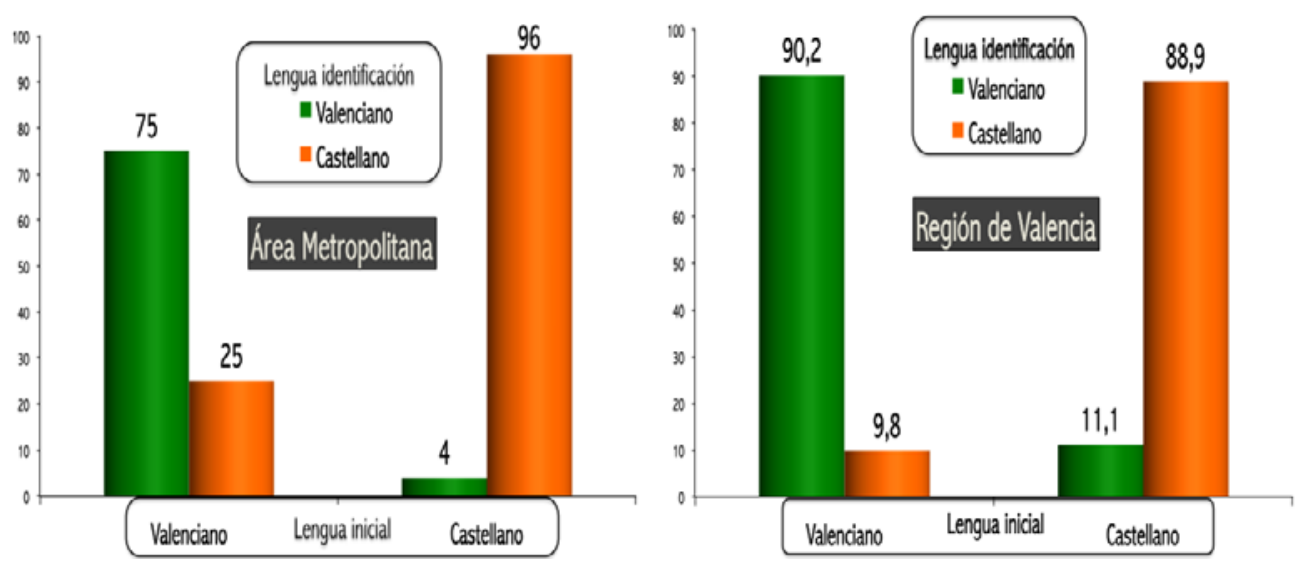

Gráfico 7. Distribución de los estudiantes de la E-2008 según L2 por lengua inicial.
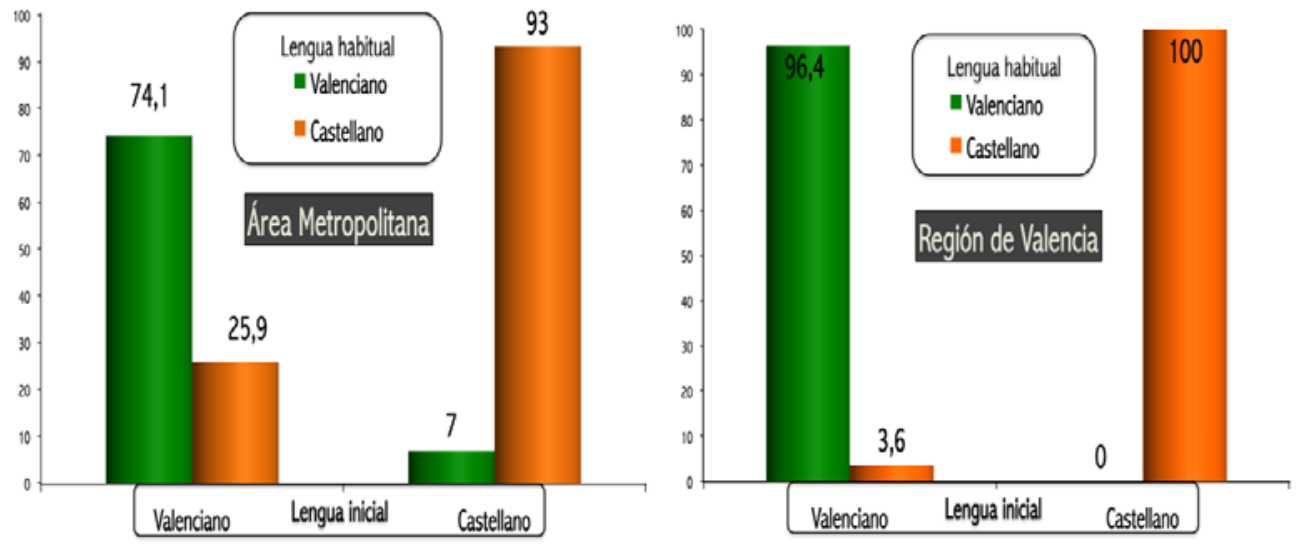

Gráfico 8. Distribución de los estudiantes de la E-2012 según L2 por lengua inicial.

pende, como en el caso anterior, de la zona de residencia: los cambios de lengua son más probables durante la adolescencia entre los estudiantes de la región de Valencia, mientras que entre los de la capital y su área metropolitana las mudas son un poco más frecuentes al iniciar una carrera universitaria. Estas mudas favorecen, además, al castellano, aunque también es cierto que aumentan ligeramente los cambios al valenciano. Podemos decir, pues, que el poder de atracción que tiene el castellano en la ciudad es tan fuerte que el abandono del valenciano puede realizarse en cualquier momento. Parece muy improbable que los jóvenes castellanohablantes de la ciudad decidan hablar valenciano habitualmente, aunque puede ocurrir $y$, con un poco más de probabilidades, tras cumplir los 20 años, durante los estudios universitarios. 


\section{Conclusiones}

Y llegamos así a responder a la cuestión sobre la existencia de nuevos hablantes o neohablantes. Recordemos que en este trabajo restringimos el término a los individuos que deciden en un momento determinado de su vida realizar una muda o cambio que favorece una lengua minorizada. Los momentos del cambio son limitados y todos ellos comportan cambios en la vida del individuo: el acceso a la primaria, la entrada en un centro de secundaria, el inicio de los estudios universitarios, de la vida laboral, el establecimiento de nuevos amigos y/o de una relación de pareja y el nacimiento de un hijo. Así mismo, la muda lingüística puede adquirir diversas formas, adoptando el uso habitual de una lengua que hasta entonces no se hablaba, cambiando la lengua que se hablaba con personas conocidas o en ámbitos concretos o cambiando las reglas de comportamiento lingüístico por las que se había decidido hablar una lengua en determinadas situaciones (Pujolar et al., 2010: 67). Por cuestiones de método en este trabajo sólo hemos estudiado el primer caso, es decir, el uso preferente del valenciano como lengua habitual. Y hemos visto que, efectivamente, algunos estudiantes de nuestra muestra ejercen este cambio. Además, no sólo hemos encontrado jóvenes neohablantes, sino que también hemos observado la continuidad de este cambio en el tiempo e incluso su ascenso, aunque sólo sea y por muy extraño que nos pueda parecer dado los bajos niveles de usos lingüísticos, en el Área metropolitana de Valencia.

Ahora bien, nuestro estudio también ha mostrado que la muda es siempre favorable al castellano y en proporciones mucho más altas. Si a ello añadimos el hecho de que la transmisión familiar del valenciano ha caído notablemente, no parecería muy razonable concluir diciendo que la supervivencia del valenciano está asegurada. Al contrario, aunque ha quedado claro que el poder de seducción del castellano es muy diferente según la zona de residencia, el valenciano está muy lejos de conseguir atraer a los jóvenes castellanohablantes en proporciones similares a como lo hace el castellano a los valencianohablantes, especialmente en el Área metropolitana.

Además, y haciendo referencia a nuestro anterior estudio (cit. 2010), constatamos que hemos llegado prácticamente a las mismas conclusiones. En primer lugar, porque los resultados de las encuestas oficiales más recientes siguen apuntando un retroceso del valenciano que empezó a mediados de los noventa y que en estos momentos parece irrefrenable y, después, porque la capacidad que tiene el castellano para seducir a los jóvenes que hablan valenciano en el ámbito familiar prácticamente cuadruplica la del valenciano. Por eso, y como preveíamos entonces, las diferencias lingüísticas entre la capital y las comarcas centrales han seguido aumentando, aunque ello no parece ser consecuencia de un incremento de neohablantes, al menos si nos atenemos a los datos de nuestras encuestas. En efecto, aunque el valenciano pierde la mínima capacidad de atracción que podía tener entre los universitarios originarios de la región de Valencia, el castellano no sale favorecido tampoco. Que el nivel de uso del valenciano de la capital y de las comarcas centrales se distancie cada vez más parece entonces atribuible a un incremento de su retroceso en la ciudad.

Un trabajo universitario de muy reciente aparición ${ }^{13}$ pronostica, basándose en dos modelos matemáticos del campo de la física y en los datos de las encuestas del SIES, que el

13 Nos referimos al trabajo de fin de grado de la estudiante Clara Miralles Vila de la Universitat de València, dirigido por Manel Perucho y del que se hicieron eco los medios de comunicación, prensa escrita y digital particularmente. 
porcentaje de valencianohablantes en el 2050 será inferior al 10\% si se mantiene la actual política lingüística. Nuestro trabajo no tiene un planteamiento prospectivo, aunque se apoya en datos longitudinales que corroboran el descenso de valencianohablantes en el ámbito familiar y el aumento de la capacidad de atracción del castellano. Y en este sentido nuestros resultados vienen a apoyar las previsiones de ese drástico retroceso del valenciano que lo situaría, siguiendo la clasificación propuesta por la UNESCO, entre las lenguas en claro peligro de extinción. Ahora bien, este pronóstico no es de obligado cumplimiento si la política lingüística del futuro gobierno valenciano da un giro brusco y toma medidas urgentes y rigurosas que vayan a favor, y no en contra, del uso del valenciano.

\section{Bibliografía}

Aguilar, C. (2001): Didàctica del català i pedagogia crítica. Castellón, Publicacions de la Universitat Jaume I.

Aparici, A. et alii. (1994): L’ús de la llengua a la Universitat. Castellón, Publicacions de la Universitat Jaume I.

AVL (Academia Valenciana de la Lengua) (2005): Llibre blanc de l'ús del valencià-I. Enquesta sobre la situació social del valencià 2004. València: AVL.

Bodoque, A. (2009): La política lingüística dels governs valencians (1983-2008). Un estudi de polítiques públiques. València, Universitat de València.

Casesnoves, R. (2005): “L'evolució del coneixement del valencià 1991-2001: una dècada de canvis socials, demogràfics i lingüístics", Journal of Catalan studies 1(1), págs. 32-51.

Casesnoves, R. (2010): “¿Qué seduce más a los jóvenes, hablar castellano o valenciano? ” En Actas del IX Congreso Internacional de Lingüistica General. Valladolid: Publicaciones de la Universidad de Valladolid, págs. 468-482.

Casesnoves, R. (2012): "El valencià en 25 anys (I): com són els seus parlants", Revista de Llengua $i$ Dret, 58: http://revistes.eapc.gencat.cat/index.php/rld/index (24-05-2015).

Casesnoves, R. (en prensa): "L'evolució del coneixement del valencià en dues dècades: els censos lingüístics del 1991 al 2011", Treballs de Sociolingüistica Catalana, 26.

Castelló, R. (2006): Els estudis sobre la situació sociolingüística al País Valencià. Barcelona, IEC: $\mathrm{http} / / / \mathrm{http}: / /$ blogs.iec.cat/cruscat/publicacions/xc/pais-valencia (24-05-2015).

Colom, F. (1998): El futur de la llengua entre els joves de València. València, Denes 10.

Fabà, A. (2005): "Llengua inicial i llengua d'identificació". En Vila, X., A. Fabà y V. Bretxa (eds.): Estadística sobre els usos lingüistics a Catalunya 2003. Barcelona, Generalitat de Catalunya, págs. 55-79.

Ferrando A. (1991): "Les perspectives de normalització lingüística al País Valencià". En J. Martí (ed.): Processos de Normalització Lingüistica: l'extensió d'ús social i de normativització. Barcelona, Columna, págs. 103-137.

Institut Valencià d'Estadística (IVE )(portal electrónico): http://www.ive.es (21-05-2015)

Marí, I. (2012): "Els usos lingüístics: tres consideracions sobre la delimitació conceptual, la consolidació i la modificabilitat", Treballs de Sociolingüistica Catalana, 22, págs. 17-26.

Montoya, B. (2008): "La transmissió familiar del valencià". En AVL (ed.): Llibre blanc de l'ús del valencià -II: iniciatives per al foment del valencià. València, Publicacions de l'Acadèmia Valenciana de la Llengua, págs. 7-25.

Montoya, B. \& A. Mas (2011): La transmissió familiar del valencià. València, AVL.

Ninyoles, R.L. (1992): "Sociología de la lengua". En García, M. (coord.): La sociedad valenciana de los 90. València, Alfons el Magnànim /Generalitat Valenciana, págs. 421-438.

Pradilla, M.A. (2008): "Vint-i-cinc anys d'ús i ensenyament del valencià". En Boix, E. (ed.): Els futurs del català. Un estat de la qüestió, una qüestió d'estat. Barcelona, Publicacions de la Universitat de Barcelona, págs. 51-80. 
Pujolar, Joan; González, Isaac; Martínez, Roger, 2010. "Les mudes lingüístiques dels joves catalans" Llengua i ús, 48: 65-75. Disponible en línea: http://www.gencat.cat/llengua (21-05-2010)

Ramallo, F. (2013): "Lenguas de España: territorios, educación, políticas e ideologías". En Moreno Fernández, F. (ed.) Las lenguas de España a debate. Valencia, Uno y Cero Ediciones, págs. 12-45. SIES (portal electrónico): http://www.edu.gva.es/polin/val/sies/sies_fonum.htm (24-05-2015)

Villaverde, J.A. (2007): "Comportament dels grups lingüístics i representacions sobre la llengua". En E. Querol (ed.): Llengua i societat als territoris de parla catalana a l'inici del segle XXI: L'Alguer, Andorra, Catalunya, Catalunya Nord, la Franja, Illes Balears i Comunitat Valenciana. Barcelona, Generalitat de Catalunya, págs. 143-181. 